\title{
Lactate Clearance Vs Revised Trauma Score
} Samir Mohamed Attia ${ }^{1}$, Rasha Rizk Elzehery ${ }^{2}$, Mohamed El-Said Ahmed ${ }^{3}$, Nourhan Hany Hassanien Mohamed ${ }^{* 4}$

Departments of ${ }^{1}$ Vascular Surgery, ${ }^{2} \mathrm{Clinical}$ Pathology, ${ }^{3} \mathrm{Critical}$ Care Medicine and ${ }^{4}$ Emergency Medicine, Faculty of Medicine, Mansoura University, Egypt

*Corresponding author: Nourhan Hany Hassanien Mohamed, Mobile: (+20)1017003699, E-Mail: nourhanhany314@ gmail.com

\begin{abstract}
Background: Trauma-related deaths are one of the top 10 causes of death. Resuscitation in trauma and critically ill patients are challenging. Clinical and laboratory parameters are used to verify the different measurements. Lactate is a diagnostic and prognostic biomarker in sepsis and trauma.

Objective: The aim of the current work was to investigate lactate clearance in patients admitted to emergency department (ED) and compare with the revised trauma score to predict mortality rate in polytrauma patients.

Patients and methods: This prospective case-control study included a total of 200 polytraumatic patients with availability of Blood sampling, admitted to Department of Emergency Medicine, Mansoura University Hospitals. This study was conducted over a period of 12 months. Serum lactate was collected in tubes before receiving any type of treatment. The analysis was performed within $1 \mathrm{~h}$ from blood collection and six hours.

Results: Regarding association with mortality, Lactate first hour, Lactate after 6 hours and Lactate clearance demonstrated significant correlation with it $(\mathrm{P}<0.05)$, while the remaining factors were not correlated $(\mathrm{P}>0.05)$. Regarding association with incidence of discharge for ICU, type of injury and Revised trauma score were correlated with it $(\mathrm{P}<0.05)$.

Conclusion: It could be concluded that serum lactate level and lactate clearance could be used as an independent predictor of mortality, incidence of discharge for ICU as well as admission to ICU with comparable efficacy to revised trauma score (RTS) in terms of Triage management.
\end{abstract}

Keywords: Revised trauma score, Lactate clearance, ICU.

\section{INTRODUCTION}

Trauma is the third overall cause of death and the first before 40 years of age, and is responsible for handicaps and high costs ${ }^{(\mathbf{1})}$. Most deaths (80\%) occurred within $48 \mathrm{~h}$ and hemorrhage continues to be one of the two leading causes of death ${ }^{(2)}$. The main principles of trauma patient care are to recognize and treat hemorrhage early, limit the consequences of shock, and diagnose traumatic lesions. Hypoperfusion is still difficult to diagnose and remains so and favors adverse inflammatory and immunologic effects, coagulopathy, development of infection and organ failures, and finally precipitates to late mortality ${ }^{(3)}$.

Several lines of evidence now attest that the most efficient management of trauma patients in the ED encompasses a timely and thoughtful analysis of the patient's situation, by means of clinical examination, diagnostic imaging and laboratory testing (4). Indeed, the availability of rapid diagnostic tests that may allow safe triage and immediate risk stratification of the single patient should be regarded as a valuable perspective for more efficient utilization of resources in short stay units such as trauma centers and emergency rooms ${ }^{(5)}$.

Several trauma scores have developed to assess the severity of trauma which is extremely important in early recognition and management of life threating conditions in trauma patients, two of the most important scores are revised trauma score (RTS) and injury severity score (ISS) ${ }^{(6)}$.

The initial surge in lactate values is known to clear over time, and numerous studies have evaluated 6-h and 12-h lactate clearance. However, the trauma surgeon needs an objective indicator of his resuscitation efforts in record time. To find if blood lactate and a 2-h lactate clearance can predict mortality in trauma and to compare these with the available scores in trauma, namely, the revised trauma score (RTS), injury severity score (ISS) and the traumarelated injury severity score (TRISS) ${ }^{(7)}$.

Several studies have determined the role of lactate elevation and its clearance as predictors of mortality and stay in the intensive care unit (ICU) in cases of sepsis, cardiopulmonary resuscitation, severe burns. Blood lactate levels above $2 \mathrm{mmol} / \mathrm{L}$ are proposed as an early and reliable marker of tissue hypoperfusion and, therefore, their measurement would be useful in critically ill patients at risk of developing shock due to any etiology. It has also been postulated that clearance of lactate levels during resuscitation may be a prognostic marker that indicates the patient's response to treatment. Persistently elevated lactate seems to be associated with mortality and prolonged hospital stay in trauma patients ${ }^{(\mathbf{8})}$.

The Revised Trauma Score is made up of three categories: Glasgow Coma Scale, systolic blood

This article is an open access article distributed under the terms and conditions of the Creative Commons Attribution (CC BY-SA) license (http://creativecommons.org/licenses/by/4.0/) 
pressure, and respiratory rate. The score range is $0-12$. In start triage, a patient with an RTS score of 12 is labeled delayed, 11 is urgent, and 3-10 is immediate ${ }^{\mathbf{9} \text {, }}$ 10).

The aim of the current work was to investigate lactate clearance in patients admitted to emergency department (ED) and compare with the revised trauma score to predict mortality rate in polytrauma patients.

\section{PATIENTS AND METHODS}

This prospective case-control study included a total of 200 polytraumatic patients with availability of Blood sampling, admitted to Department of Emergency Medicine, Mansoura University Hospitals. This study was conducted over a period of 12 months.

Serum lactate was collected in tubes before receiving any type of treatment. The analysis was performed within $1 \mathrm{~h}$ from blood collection and six hours.

Inclusion criteria: All patients with polytrauma aged more than 18 years and revised trauma score $\geq 11$ and this study doesn't't pregnant females.

Exclusion criteria: Advanced liver disease, renal failure, heart failure and severe anemia $\mathrm{HB}<7.0$.

\section{Methods}

1. Primary survey and resuscitation:

- A = Airway opening and maintenance of airway.

- $\quad B=$ Breathing and ventilation.

- $\mathbf{C}=$ Circulation.

- $\mathbf{D}=$ Disability: neurological status and Glasgow coma scale (GCS).

- $\mathbf{E}=$ Exposure: segmental exposure.

2. Secondary survey (from head to nail examination: head, neck, chest, abdomen, pelvis, lower limb, upper limb) and Vital sign (pulse, blood pressure, respiratory rate).

\section{AMPLE History:}

- $\mathbf{A}=$ Allergies.

- $\mathbf{M}=$ Medication currently used.

- $\mathbf{P}=$ Past illnesses / Pregnancy.

- $\mathbf{L}=$ Last meal.

- $\mathbf{E}=$ Events / Environment related to injury (Exactly what happened?).

Entire patients were subjected to:

- Full history taking, which included age, sex, occupation, and smoking habits.

- Physical examination which included: Assessment of Vital signs: (pulse, blood pressure, temperature and respiratory rate). Glasgow coma scale (GCS) (as shown in table 1) ${ }^{(11)}$.

Table (1): Glasgow coma scale (GCS) ${ }^{(11)}$.

\begin{tabular}{|c|c|c|c|c|c|}
\hline Eye opening $(E)$ & Score & Verbal response (V) & Score & Motor response (M) & Score \\
\hline - & & - & & Obeys commands & 6 \\
\hline - & & Orientated & 5 & Localises to pain & 5 \\
\hline Spontaneously & 4 & Confused & 4 & Flexion/withdrawal to pain & 4 \\
\hline To speech & 3 & Inappropriate words & 3 & Abnormal flexion & 3 \\
\hline To pain & 2 & Incomprehensible sounds & 2 & Extends & 2 \\
\hline None & 1 & None & 1 & None & 1 \\
\hline
\end{tabular}

Laboratory investigations: Serum lactate level.

Radiological investigations: Chest X- Ray, FAST abdominal ultrasonography, brain non contrast computed tomography, LSS \& cervical X-ray, pelvis X-ray and limb X- ray.

D Outcomes: Mortality within one week and ICU admission. 


\section{Ethical consideration:}

The research approval of the study was obtained from IRB of Faculty of Medicine at Mansoura University before starting the study. The researcher clarified the objective and aim of the study to the subjects included in the study. The researcher assured maintaining anonymity and confidentiality of subjects' data. Subjects were informed that they allowed to choose to participate or not in the study and that, they had the right to withdraw from the study at any time without giving any reasons. Ethics, values, culture, and beliefs of subjects were respected.

\section{Statistical analysis and data interpretation}

The collected data were coded, processed and analyzed using the SPSS (Statistical Package for Social Sciences) version 22 for Windows ${ }^{\circledR}$ (IBM SPSS Inc, Chicago, IL, USA). Data were tested for normal distribution using the Shapiro Walk test. Qualitative data were represented as frequencies and relative percentages. Chi square test $(\chi 2)$ to calculate difference between two or more groups of qualitative variables. The diagnostic performance of a test, or the accuracy of a test to discriminate diseased cases from nondiseased cases is evaluated using Receiver Operating Characteristic (ROC) curve analysis. Sensitivity and Specificity were detected from the curve and PPV,NPV and accuracy were calculated through cross tabulation.
Quantitative data were expressed as mean \pm SD (Standard deviation). Independent samples t-test was used to compare between two independent groups of normally distributed variables (parametric data). $\mathrm{P}$ value $<0.05$ was considered significant.

\section{RESULTS}

Table (2) shows the demographic characteristics of the studied cases, in which the mean age was $44.53 \pm 12.15$ years with male to female $(\mathrm{M} / \mathrm{F})$ ratio was $61.5 / 38.5$

Table (2): Demographic characteristics of the studied cases.

\begin{tabular}{|l|l|l|}
\hline \multicolumn{2}{|l|}{$\mathbf{N}=\mathbf{2 0 0}$} \\
\hline Age/years & \multicolumn{2}{|c|}{} \\
Mean \pm SD & $44.53 \pm 12.15$ \\
\hline Sex: N $(\%)$ & $\mathbf{N}$ & $\mathbf{\%}$ \\
\cline { 2 - 3 } Male & 123 & 61.5 \\
Female & 77 & 38.5 \\
\hline
\end{tabular}

Table (3) illustrates the association between demographic, vital signs and laboratory findings and mortality among studied cases. Types of injury, GCS Revised trauma score $(11,12)$, Lactate first hour, Lactate after 6 hours and Lactate clearance demonstrated significant correlation with mortality $(\mathrm{P}<0.05)$, while the remaining factors not $(\mathrm{P}>0.05)$.

Table (3): Association between demographic, vital signs and laboratory findings and mortality among studied cases.

\begin{tabular}{|l|l|l|l|}
\hline \multicolumn{1}{|l|}{ Survived } & \multicolumn{1}{l|}{ Died } \\
\hline Age/years & $44.74 \pm 12.05$ & $43.90 \pm 12.54$ & $\mathrm{p}=0.673$ \\
\hline Sex & & & \\
Male & $96(64.0)$ & $27(54.0)$ & $\mathrm{p}=0.208$ \\
Female & $54(36.0)$ & $23(46.0)$ & \\
\hline Mode of trauma & & & \\
Fator vehicle collision & $105(70.0)$ & $38(76.0)$ & $\mathrm{P}=0.821$ \\
Blunt trauma to abdomen, chest, face & $31(20.7)$ & $8(16.0)$ & \\
Assaults & $12(8.0)$ & $3(6.0)$ & \\
\hline Types of injury & $2(1.3)$ & $1(2.0)$ & \\
Orthopedic & & & $\mathrm{P}<0.001^{*}$ \\
Neurosurgical & $140(93.3)$ & $17(34.0)$ & \\
Cardiothoracic & $3(2.0)$ & $4(8.0)$ & $\mathrm{P}=0.03^{*}$ \\
Abdominal & $7(4.7)$ & $7(14.0)$ & \\
\hline GCS & $0(0.0)$ & $22(44.0)$ & $\mathrm{p}<0.001^{*}$ \\
\hline Revised trauma score & $13.07 \pm 1.62$ & $12.54 \pm 0.99$ & \\
11 & & & $\mathrm{p}<0.001^{*}$ \\
12 & $79(52.7)$ & $46(92.0)$ & $\mathrm{p}=0.002^{*}$ \\
\hline Lactate (mmol/L) first hour & $71(47.3)$ & $4(8.0)$ & $\mathrm{p}<0.001^{*}$ \\
\hline Lactate (mmol/L) after 6 hours & $8.59 \pm 3.95$ & $4.78 \pm 3.62$ & \\
\hline Lactate clearance & $2.54 \pm 1.11$ & $3.19 \pm 0.25$ & \\
\hline
\end{tabular}

All parameters described as mean \pm SD and number \& percentage

MC:Monte Carlo test , $\mathrm{t}$ :Student $\mathrm{t}$ test, Z:Mann Whitney U test $\chi^{2}=$ Chi-Square test *statistically significant (if $\mathrm{p}<0.05$ ).

Table (4) illustrates the association between demographic, vital signs and laboratory findings and incidence of admission to ICU among studied cases. Age, sex, Types of injury, RR, DBP, SBP, Revised trauma score $(11,12)$, 
Lactate first hour, Lactate after 6 hours and Lactate clearance demonstrated significant correlation with admission to ICU $(\mathrm{P}<0.05)$, while the remaining factors not $(\mathrm{P}>0.05)$.

Table (4): Association between demographic, vital signs and laboratory findings and incidence of admission to ICU among studied cases:

\begin{tabular}{|c|c|c|c|}
\hline & No admission to ICU & Admitted to ICU & test of significance \\
\hline Age/years & $47.40 \pm 11.79$ & $42.98 \pm 12.09$ & $\mathrm{p}=0.014^{*}$ \\
\hline $\begin{array}{l}\text { Sex } \\
\text { Male } \\
\text { Female }\end{array}$ & $\begin{array}{l}51(72.9) \\
19(27.1)\end{array}$ & $\begin{array}{l}72(55.4) \\
58(44.6)\end{array}$ & $\mathrm{p}=0.015^{*}$ \\
\hline $\begin{array}{l}\text { Mode of trauma } \\
\text { Motor vehicle collision } \\
\text { Fall from height } \\
\text { Blunt trauma to abdomen, } \\
\text { chest, face } \\
\text { Assaults } \\
\end{array}$ & $\begin{array}{l}51(72.9) \\
15(21.4) \\
4(5.7) \\
0(0.0)\end{array}$ & $\begin{array}{l}92(70.8) \\
24(18.5) \\
11(8.5) \\
3(2.3) \\
\end{array}$ & $\mathrm{P}=0.511$ \\
\hline $\begin{array}{l}\text { Types of injury } \\
\text { Orthopedic } \\
\text { Neurosurgical } \\
\text { Cardiothoracic } \\
\text { Abdominal } \\
\end{array}$ & $\begin{array}{l}66(94.3) \\
0(0.0) \\
4(5.7) \\
0(0.0)\end{array}$ & $\begin{array}{l}91(70.0) \\
7(5.4) \\
10(7.7) \\
22(16.9)\end{array}$ & $\mathrm{P}<0.001 *$ \\
\hline GCS & $13.05 \pm 1.43$ & $12.87 \pm 1.55$ & $\mathrm{p}=0.402$ \\
\hline $\mathbf{R R}$ & $21.70 \pm 3.56$ & $23.81 \pm 3.38$ & $\mathrm{p}<0.001 *$ \\
\hline Systolic blood pressure & $125.50 \pm 9.54$ & $118.58 \pm 15.87$ & $\mathrm{p}=0.001^{*}$ \\
\hline HR & $87.27 \pm 8.56$ & $77.23 \pm 11.29$ & $\mathrm{p}=0.188$ \\
\hline Diastolic blood pressure & $81.63 \pm 9.49$ & $83.53 \pm 9.82$ & $\mathrm{p}<0.001 *$ \\
\hline $\begin{array}{l}\text { Revised trauma score } \\
11 \\
12\end{array}$ & $\begin{array}{l}29(41.4) \\
41(58.6)\end{array}$ & $\begin{array}{l}96(73.8) \\
34(26.2)\end{array}$ & $\mathrm{p}<0.001 *$ \\
\hline Lactate (mmol/L) first hour & $9.82 \pm 3.89$ & $6.47 \pm 3.89$ & $\mathrm{p}<0.001^{*}$ \\
\hline $\begin{array}{l}\text { Lactate }(\mathrm{mmol} / \mathrm{L}) \text { after } 6 \\
\text { hours }\end{array}$ & $2.35 \pm 0.84$ & $2.89 \pm 1.48$ & $\mathrm{p}=0.005^{*}$ \\
\hline Lactate clearance & $0.709 \pm 0.18$ & $0.428 \pm 0.326$ & $\mathrm{p}<0.001 *$ \\
\hline
\end{tabular}

all parameters described as mean \pm SD and number \& percentage.MC:Monte Carlo test , $\mathrm{t}$ :Student $\mathrm{t}$ test , Z:Mann Whitney $\mathrm{U}$ test $\chi 2=$ Chi-Square test $*$ statistically significant (if $\mathrm{p}<0.05$ )

Table (5) displayed that, Revised trauma score could be used as a predictor of mortality among studied and its sensitivity, specificity, PPV, NPV and accuracy were 92.0, 47.3, 36.8, 94.7 and 58.5 respectively. Revised trauma score could be used as a predictor of ICU admission among studied and its sensitivity, specificity, PPV, NPV and accuracy were $73.8,58.6,76.8,54.7$ and 68.5 respectively.

Table (5): Validity of revised trauma score in predicting mortality and ICU admission among studied cases:

\begin{tabular}{|l|l|l|l|l|l|}
\hline \multicolumn{2}{|c|}{$\begin{array}{l}\text { Sensitivity } \\
(\%)\end{array}$} & $\begin{array}{l}\text { Specificity } \\
(\%)\end{array}$ & PPV(\%) & \multicolumn{1}{l|}{ NPV(\%) } & \multicolumn{1}{c|}{ Accuracy(\%) } \\
\hline $\begin{array}{l}\text { Revised trauma score } \\
\text { of mortality }\end{array}$ & 92.0 & 47.3 & 36.8 & 94.7 & 58.5 \\
\hline $\begin{array}{l}\text { Revised trauma score } \\
\text { of ICU admission }\end{array}$ & 73.8 & 58.6 & 76.8 & 54.7 & 68.5 \\
\hline
\end{tabular}

AUC: Area under curve $\quad$ PPV: Positive predictive value NPV: Negative predictive value

Table (6) displayed that, lactate clearance could be used as a valid predictor of ICU admission among studied cases $(\mathrm{P}<0.001)$. At cut off 0.4616 (AUC=0.832), its sensitivity, specificity, PPV, NPV and accuracy were 88.0, 88.0, 71.0, 95.7 and 88.0 respectively. Lactate clearance could be used as a valid predictor of ICU admission among studied cases $(\mathrm{P}<0.001$ ). At cut off 0.6290 (AUC=0.743), its sensitivity, specificity, PPV, NPV and accuracy were 63.8, 58.6, $74.1,46.6$ and 62 respectively. 
Table (6): Validity of lactate clearance in predicting mortality and ICU admission among studied cases.

\begin{tabular}{|l|l|l|l|l|l|l|l|l|}
\hline \multicolumn{2}{|c|}{$\begin{array}{l}\text { AUC } \\
\text { (95\% } \\
\text { CI) }\end{array}$} & \multicolumn{2}{|c|}{$\begin{array}{l}\text { P value off } \\
\text { point }\end{array}$} & $\begin{array}{l}\text { Sensitivity } \\
(\%)\end{array}$ & $\begin{array}{l}\text { Specificity } \\
(\%)\end{array}$ & $\begin{array}{l}\text { PPV } \\
(\%)\end{array}$ \\
\hline $\begin{array}{l}\text { Lactate clearance } \\
\text { of mortality }\end{array}$ & $\begin{array}{l}0.832 \\
(0.757- \\
0.908)\end{array}$ & $0.001 *$ & 0.4616 & 88.0 & 88.0 & 71.0 & 95.7 & 88.0 \\
\hline $\begin{array}{l}\text { Lactate clearance } \\
\text { of mortality of } \\
\text { ICU admission }\end{array}$ & $\begin{array}{l}0.743 \\
(0.676- \\
0.811)\end{array}$ & $0.001 *$ & 0.6290 & 63.8 & 58.6 & 74.1 & 46.6 & 62.0 \\
\hline
\end{tabular}

AUC: Area under curve $\quad$ PPV: Positive predictive value $\quad$ NPV: Negative predictive value

Table (7) demonstrated that, Revised trauma score 11, 12 and Creatinine clearance were demonstrated to be significant predictors of mortality among studied cases. Revised trauma score 11, 12 and Creatinine clearance were demonstrated to be significant predictors of ICU admission among studied cases.

Table (7): Predictors of mortality and ICU admission among studied cases

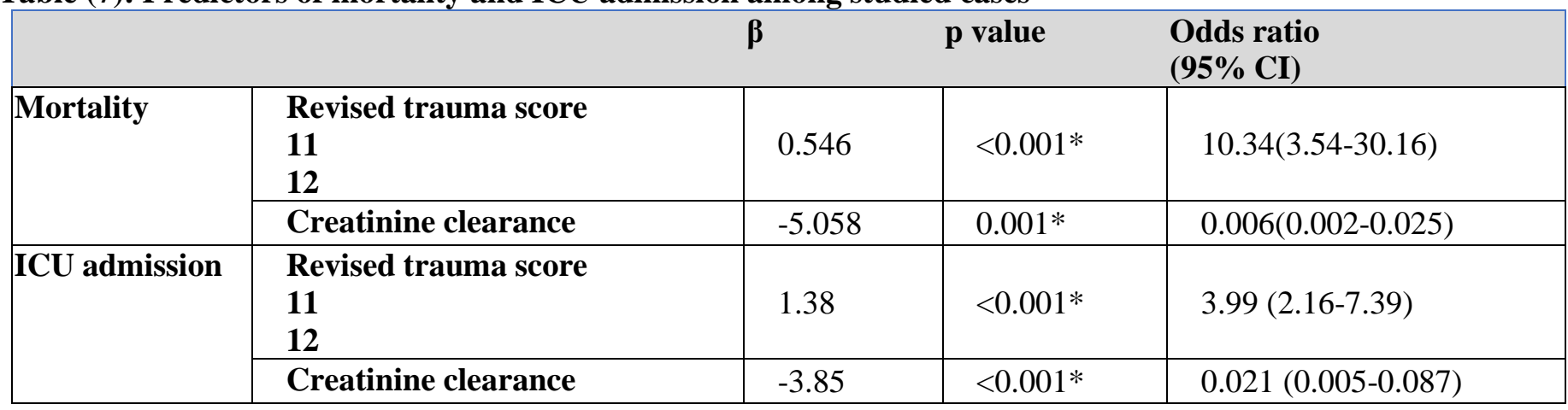

\section{DISCUSSION}

This prospective case-control study was carried out on subjects aged more than 18 years and revised trauma score $\geq 11$ admitted to Department of Emergency Medicine, Mansoura University Hospitals, over 12 months on 200 polytrauma patients.

In terms of the demographic data, the current study displayed that, the mean age was $44.53 \pm 12.15$ years with male to female $(\mathrm{M} / \mathrm{F})$ ratio was $61.5 / 38.5$. Concerning mode of trauma, the current study demonstrated that motor vehicle collision was the most common mode of trauma ( $71.5 \%$ ) followed by fall from height (19.5\%), then blunt trauma to abdomen, chest and face (7.5\%) and lastly assaults (1.5\%). In accordance, Odom et $\boldsymbol{a l} .{ }^{(12)}$ demonstrated that, motor vehicle collision was the most common mode of trauma followed by falls and lastly penetrating injuries.

Regarding type of injury, Orthopedic was the most common type of injury (78.5\%), followed abdominal $(11 \%)$ by then cardiothoracic (7\%) and lastly neurosurgical (3.5\%). While, Odom et al. ${ }^{(12)}$ demonstrated that, head and face were the most common type of injury, followed by chest and abdomen by and lastly extremities.

Regarding association with mortality, types of injury, GCS Revised trauma score $(11,12)$, Lactate first hour, Lactate after 6 hours and Lactate clearance demonstrated significant correlation with mortality $(\mathrm{P}<0.05)$, while the remaining factors not $(\mathrm{P}>0.05)$. Regarding association with incidence of discharge for ICU, Type of injury and Revised trauma score (11 and
12) were demonstrated to have a significant correlation with incidence of discharge for ICU $(\mathrm{P}<0.05)$, while the other parameters not. Lactate first hour, Lactate after 6 hours and Lactate clearance demonstrated significant correlation with admission to ICU $(\mathrm{P}<0.05)$, while the remaining factors not $(\mathrm{P}>0.05)$.

Odom et al. ${ }^{(12)}$, Chana et al. ${ }^{(13)}$, Caputo et al. ${ }^{(14)}$ and Mutschler et al. ${ }^{(15)}$ reported that patients with high blood lactate have higher risk of death compared to those with levels within the normal laboratory range. The clearance of lactate may represent good parameter to analyze the quality of resuscitation measures in trauma and information on prognosis, especially in early mortality. Thus, the lactate can be used as a marker between the demand and availability of oxygen and its level changes can be used as effective marker in resuscitation maneuvers, even in patients with stability in vital signs.

In the same line, Cureton et al. ${ }^{(16)}$ conducted their study on head trauma cases to assess the correlation between admission lactate and both survival and neurologic function. They demonstrated that, increased lactate was associated with more severe head injury $(\mathrm{P}<0.0001)$. The admission lactate was $2.2+/-$ $0.07,3.7+/-0.7$, and $4.7+/-0.8 \mathrm{mmol} / \mathrm{L}$ in patients with mild, moderate, and severe head injury respectively $(\mathrm{P}<0.01)$.

Of note, since lactate is a readily available fuel source of the injured brain, this may be a mechanism by which brain function is preserved in trauma patients. Elevations in lactate due to anaerobic metabolism in 
trauma patients may have beneficial effects by protecting the brain during injury ${ }^{(\mathbf{1 6})}$.

The present study have found a complex relationship between blood pressure, lactate, and mortality. Most importantly, such results confirm the concept of "occult hypoperfusion": normotensive patients with elevated lactates are at higher risks of death. Similar findings have been observed in brain injured patients ${ }^{(17)}$ and even in community-dwelling adults ${ }^{(18)}$.

This suggests that our finding may have physiologic relevance, although the mechanism remains unclear and could be considered as an avenue for future investigation.

This came in accordance with Odom et al. ${ }^{(12)}$ who conducted their study on 4,742 trauma patients who had an initial lactate measured during a 10 -year period. They demonstrated that, Initial lactate level was strongly correlated with mortality: when lactate was less than $2.5 \mathrm{mg} / \mathrm{dL}, 5.4 \%$ (95\% confidence interval [CI], 4.5-6.2\%) of patients died; with lactate $2.5 \mathrm{mg} / \mathrm{dL}$ to $4.0 \mathrm{mg} / \mathrm{dL}$, mortality was $6.4 \%$ (95\% CI, $5.1-7.8 \%)$; with lactate $4.0 \mathrm{mg} / \mathrm{dL}$ or greater, mortality was $18.8 \%$ (95\% CI, 15.7-21.9\%).

Similarly, Régnier et al. ${ }^{\left({ }^{19)}\right.}$ demonstrated that, blood lactate levels at admission were elevated in 327 $(56 \%)$ patients. The lactate clearance should be calculated within the first $2 \mathrm{~h}$ after admission as LCO-2 $\mathrm{h}$ was correlated with $\mathrm{LC} 0-4 \mathrm{~h}(\mathrm{R}=0.55, \mathrm{P}<0.001)$ but not with LC2-4 h ( $\mathrm{R}=0.04$, not significant). The lactate clearance provides additional predictive information to initial blood lactate levels and triage scores and the reference score.

Furthermore, Callaway et al. ${ }^{(20)}$ revealed that, lactate was associated with significantly increased mortality in normotensive elderly blunt trauma patients as compared with the normal lactate group; patients in the severely elevated lactate group had 4.2 increased odds of death. However, because of the high baseline mortality rates in elderly trauma patients, "normal" lactate does not offer complete reassurance to the clinician.

On the contrary, Pal et al. (21) displayed that, admission lactate levels failed to show useful predictive accuracy for hospital death. Serum lactate levels need not be obtained routinely but can be reserved for patients who will be admitted to the intensive care unit and/or require an emergency operation.

In addition, a study by Freitas and Franzon (22) did not find any correlation between admission lactate and mortality in patients treated with multiple traumas.

Moreover, DSouza et al. ${ }^{(7)}$ demonstrated that, independently collected 2-h lactate could predict mortality (values $>2.5 \mathrm{mmol} / \mathrm{L}$ ) (sensitivity of 60 and specificity of 87.57). Two-hour lactate clearance had no statistical significance in predicting mortality in trauma patients but maybe considered to be an important prognostic aid to predict adequacy of resuscitation efforts.

In terms of the relation between lactate and admission, Heinonen et al. ${ }^{(23)}$ came in agreement with the current study as they demonstrated that, 48-h lactate clearance to be of prognostic significance, which may be of value in the critical care admission setting and not necessarily in an emergency setup. John et al. (24) postulated that a prehospital lactate could be useful in risk-stratifying normotensive trauma patients.

Thus, We draw attention to a systematic review by Baxter et al. (25), which found that elevated emergency department lactate levels are associated with mortality and concluded that lactate is a useful marker of outcome in trauma and that the potential roles of serial lactate measurement and lactate clearance require further research, a finding similar to ours.

Revised trauma score could be used as a predictor of ICU admission among studied and its sensitivity, specificity, PPV, NPV and accuracy were 92.0, 47.3, 36.8, 94.7 and 58.5 respectively. The current study demonstrated that, Revised trauma score $(11,12)$ had comparable efficacy to lactate as triage systems.

In accordance, DSouza et al. ${ }^{(7)}$ demonstrated that, Injury severity score is comparable to lactate as a triage score.

The trauma-related injury severity score and revised trauma scores were not found to be good indicators of 30-day hospital mortality. A new trauma score (NTS) using GCS score, SBP and arterial saturation of oxygen has been found to be superior to RTS in a study by Jeong et al. ${ }^{(26)}$.

With regard to lactate clearance, the current study revealed that, lactate clearance could be used as a valid predictor of ICU admission among studied cases $(\mathrm{P}<0.001)$. At cut off 0.4616 ( $\mathrm{AUC}=0.832$ ), its sensitivity, specificity, PPV, NPV and accuracy were $88.0,88.0,71.0,95.7$ and 88.0 respectively.

The current study demonstrated that, lactate clearance could be used as a valid predictor of ICU admission among studied cases $(\mathrm{P}<0.001)$. At cut off 0.4616 (AUC=0.832), its sensitivity, specificity, PPV, NPV and accuracy were 88.0, 88.0, 71.0, 95.7 and 88.0 respectively.

This came in agreement with Husain et al. ${ }^{(27)}$ who demonstrated that, lactate clearance time may be used to predict mortality and is associated with outcome at discharge.

On the contrary, Freitas and Franzon ${ }^{(22)} \mathrm{did}$ not find any correlation between lactate clearance and mortality in patients treated with multiple traumas.

Dezman et al. ${ }^{(28)}$ concluded that serial lactate measurements predict mortality in trauma and not lactate clearance.

In addition, Altunbas et al. ${ }^{(29)}$ felt that, 2-h lactate clearance may not be sufficient to predict 30day mortality in trauma patients. 
Of note, On many levels (physiologic, mechanistic, and outcome measures), the current study demonstrated that the patients who did not have lactate measured were less severely injured than those who did, and despite this, lactate and its clearance remain a strong predictor of mortality.

\section{CONCLUSION}

It could be concluded that serum lactate level and lactate clearance could be used as an independent predictor of mortality, incidence of discharge for ICU as well as admission to ICU with comparable efficacy to RTS in terms of Triage management.

\section{REFERENCES}

1. Murray C, Lopez A (1997): Mortality by cause for eight regions of the world: Global Burden of Disease Study. Lancet, 117:1269-76

2. Sauaia A, Moore F, Moore E et al. (1995): Epidemiology of trauma deaths: A reassessment. J Trauma, 38:185-93

3. Claridge J, Crabtree T, Pelletier S et al. (2000): Persistent occult hypoperfusion is associated with a significant increase in infection rate and mortality in major trauma patients. $\mathbf{J}$ Trauma, 48:8-14.

4. Mikkelsen M, Miltiades A, Gaieski D et al. (2009): Serum lactate is associated with mortality in severe sepsis independent of organ failure and shock. Crit Care Med., 37:1670-1677.

5. Nguyen H, Rivers E, Knoblich B et al. (2004): Early lactate clearance is associated with improved outcome in severe sepsis and septic shock. Critical Care Medicine, 32(8): 16371642.

6. Wong S, Leung G (2008): Injury Severity Score (ISS) vs. ICD-derived Injury Severity Score (ICISS) in a patient population treated in a designated Hong Kong trauma centre. McGill Journal of Medicine, 11(1): 9-13.

7. DSouza D, Sunny A, Sima $R$ et al. (2020): Lactate Measurement in Critically Injured-Prognostic Importance. Indian Journal of Surgery, 20: 1-5.

8. Morales C, Ascuntar A, Londoño J et al. (2019): Lactate clearance: Prognostic mortality marker in trauma patients. March 2019Colombian Journal of Anesthesiology, 47(1):41 48.

9. Wilbur T, Donald V (2009): Taber's cyclopedic medical dictionary. F a Davis Co., Pp. 2366. ISBN 978-0-8036-15595. https://www.amazon.com/Tabers-Cyclopedic-MedicalDictionary-Version/dp/0803659040

10. Champion H, Sacco W, Copes W et al. (1989): A revision of the Trauma Score. The Journal of Trauma, 29 (5): 623-9.

11. Sternbach G (2000): The Glasgow coma scale. The Journal of Emergency Medicine, 19(1): 67-71.

12. Odom S, Howell M, Silva G et al. (2013): Lactate clearance as a predictor of mortality in trauma patients. Journal of Trauma and Acute Care Surgery, 74(4): 999-1004.

13. Chana M, Manson J, Davenport $\mathrm{R}$ et al. (2012): Time course of lactate clearance in trauma and its relevance to outcomes. Scandinavian Journal of Trauma, Resuscitation and Emergency Medicine, 20(1): 1-1.

14. Caputo N, Fraser R, Paliga A et al. (2013): Triage vital signs do not correlate with serum lactate or base deficit, and are less predictive of operative intervention in penetrating trauma patients: a prospective cohort study. Emergency Medicine Journal, 30(7): 546-550.

15. Mutschler M, Nienaber U, Brockamp T et al. (2013): Bouillon 1 B, Maegele M, TraumaRegister DGU: Renaissance of base deficit for the initial 2 assessment of trauma patients: a base deficit-based classification for 3 hypovolemic shock developed on data from 16,305 patients derived from the 4 Trauma Register DGU (R). Crit Care, 17(2): 42-48.

16. Cureton E, Kwan R, Dozier K et al. (2010): A different view of lactate in trauma patients: protecting the injured brain. Journal of Surgical Research, 159(1): 468-473.

17. Butcher I, Maas A, Lu J et al. (2007): Prognostic value of admission blood pressure in traumatic brain injury: results from the IMPACT study. Journal of Neurotrauma, 24(2): 294-302.

18. Okumiya K, Matsubayashi K, Wada T et al. (1999): AUshaped association between home systolic blood pressure and four- year mortality in community- dwelling older men. Journal of the American Geriatrics Society, 47(12): 14151421.

19. Régnier M, Raux M, Le Manach Y et al. (2012): Prognostic significance of blood lactate and lactate clearance in trauma patients. The Journal of the American Society of Anesthesiologists, 117(6): 1276-1288.

20. Callaway D, Shapiro N, Donnino M et al. (2009): Serum lactate and base deficit as predictors of mortality in normotensive elderly blunt trauma patients. Journal of Trauma and Acute Care Surgery, 66(4): 1040-1044.

21. Pal J, Victorino G, Twomey $P$ et al. (2006): Admission serum lactate levels do not predict mortality in the acutely injured patient. Journal of Trauma and Acute Care Surgery, 60(3): 583-589.

22. Freitas A, Franzon O (2015): Lactate as predictor of mortality in polytrauma. ABCD. Arquivos Brasileiros de Cirurgia Digestiva (São Paulo), 28(3): 163-166.

23. Heinonen E, Hardcastle T, Barle $H$ et al. (2014): Lactate clearance predicts outcome after major trauma. African Journal of Emergency Medicine, 4(2): 61-65.

24. John A, McCoy A, Moyes A et al. (2018): Prehospital lactate predicts need for resuscitative care in non-hypotensive trauma patients. Western Journal of Emergency Medicine, 19(2): 224-29.

25. Baxter J, Cranfield K, Clark G et al. (2016): Do lactate levels in the emergency department predict outcome in adult trauma patients? A systematic review. Journal of Trauma and Acute Care Surgery, 81(3): 555-566.

26. Jeong J, Park Y, Kim D et al. (2017): The new trauma score (NTS): a modification of the revised trauma score for better trauma mortality prediction. BMC Surgery, 17(1): 1-9.

27. Husain F, Martin M, Mullenix $P$ et al. (2003): Serum lactate and base deficit as predictors of mortality and morbidity. The American Journal of Surgery, 185(5): 485491.

28. Dezman Z, Comer A, Smith G et al. (2018): Repeat lactate level predicts mortality better than rate of clearance. The American Journal of Emergency Medicine, 36(11): 20052009.

29. Altunbas E, Omercikoglu S, Akoglu H et al. (2018): Prognostic value of 2-hour lactate level and lactate clearance for 30-day mortality and comparison with trauma scores in multi-trauma patients. Pakistan Journal of Medical Sciences, 34(3): 676. 